\section{Brainstem Death due to hypertension induced brain hemorrhage}

\author{
Satyajit Patra* and Sreeraj Suresh \\ American International Medical University, Saint Lucia
}

\section{Introduction}

Brain stem death is when a person has permanently lost the potential for consciousness and the capacity to breathe. When this happens, a machine called a ventilator keeps oxygen circulating through the person's bloodstream - a person confirmed as being dead when their brain stem function is permanently lost [1].

Untreated high blood pressure is a major preventable cause of brain hemorrhages, Aneurysm is a weakening in a blood vessel wall and causes swelling in the vessels. It can burst and bleed into the brain, leading to a stroke. High blood pressure is a common and dangerous condition. About 1 of 3 U.S. adults or about 75 million people have high blood pressure [2]. Only about half (54\%) of these people have their high blood pressure under control. Brain stem strokes can have complex symptoms, and they are difficult to diagnose. A person may have vertigo, dizziness, and severe imbalance without the hallmark of most strokes weakness on one side of the body. The symptoms of vertigo dizziness or imbalance usually occur together; dizziness alone is not a sign of stroke [3]. Brain stem stroke can also cause double vision, slurred speech, and decreased the level of consciousness. The brain stem, a half inches structure, controls all basic activities of the central nervous system like consciousness, blood pressure, and breathing. All of the motor control for the body flows through it. Brain stem strokes can impair any or all of these functions. These complications are often predictable and, with prompt recognition, can be treated. If complications are dealt with quickly, there is a good chance of recovery [4].

More severe brain stem strokes can cause the locked-in syndrome, a condition in which survivors can move only their eyes. If a stroke in the brain stem results from a clot, the faster blood flow can be restored in this critical area, the better the chances for recovery [5]. The public and healthcare professionals must know all of the symptoms of a stroke and are aware that some brain stem strokes heave distinct symptoms. Patients need to receive treatment as soon as possible to promote the best recovery. Hypertensive crises are important medical emergencies, which may result

\section{More Information}

*Address for Correspondence: Satyajit Patra, Associate Dean, Student Affairs, Director of Research and PhD Program, Associate Professor, Division of Biochemistry and Genetics, American International Medical University, Beausejour Road, North Entrance, Gros Islet, Saint Lucia, Fax: 1-758-450-0138, Tel: 1-758-4500130/ext: 2239; Email: dr.patra@aimu-edu.us; satyajitpatra@gmail.com

Submitted: 10 October 2018 Approved: 06 December 2019 Published: 09 December 2019

How to cite this article: Patra S, Suresh S. Brainstem Death due to hypertension induced brain hemorrhage. J Clin Intensive Care Med. 2019; 4: 042-043.

DOI: dx.doi.org/10.29328/journal.jcicm.1001025

Copyright: Patra S, et al. This is an open access article distributed under the Creative Commons Attribution License, which permits unrestricted use, distribution, and reproduction in any medium, provided the original work is properly cited

\section{W) Check for updates}

in acute end-organ injuries such as stroke, pulmonary edema, congestive heart failure, aortic dissection, acute myocardial infarction, unstable angina, acute renal failure, and hypertensive encephalopathy [6]. Using a patient's case study, we would know how a patient presents with this condition and also more about the risk factor and cause of this condition.

\section{Case}

Here is a case of a 62-year-old patient, referred case from VSM, Hospital with c/o of numbness over the right upper limb and head A/w weakness since morning 6 AM. Initially managed in the local hospital, the patient was intubated and ventilated in the emergency room, given a low GCS score of E1V1M3. A permanent pacemaker in situ is in place since 2015 at Amrita Institute of Medical Sciences and Research Centre, Kochi. The patient has a known c/o hypertension; the patient was on clopidogrel and ecosprin since 2015. On arrival, the patient was intubated and ventilated on arrival to the hospital; vitals were as follows PR-112 bpm, BP-200/100 $\mathrm{mm} / \mathrm{hg}$, RR-22, afebrile, CVS-112bpm, CNS-E1V1M2, PUPIL-B/1 PINPOINT, P/A-soft, and Chest examinationAEBE+. Immediate CT brain showed large brain stem bleed. Examined as a very critical condition and explained to patient bystanders about the need for continued ventilation, repeat CT brain performed. The patient received with Inj. MANNITOL 100 ml 1-1-1, Inj. ROME 1 gm 1-1-1, Inj. FOSPHEN 
150 1-1-1, Inj. LEVIPIL 500 1-1-1, Inj. PANTOP 40 1-0-1, Inj. EMESET 4 gm 1-1-1, Inj. Vitamin K OD, Inj. ETHAMSYLATE 250 1-1-1, Inj. DEXONA 4 mg 1-1-1, Inj. NIMODIP $1 \mathrm{mg} / \mathrm{hr}$, DIMOX 250 1-1-1. Cardiology and nephrology consultation was recommended. On repeat CT Brain report no increase in bleed. 16/12 E1M2 Vet, K+ -5.3, FBS - 129, added tab Cilacar 10 mg 1-1-1, tab Acidose 1-1-1, tab Nephrosave 1-11.17/12 E1M3 Vet Weak spontaneous respiration, Slight improvement explained but guarded prognosis. 18/12 T piece trail done but poor respiratory efforts. Poor prognosis explained Creatine levels were 3.4, Urea - 93. 19/12 E1M1Vet RR 18/min weak. 20/12 E1M3 Cough reflex+, Sputum C\&S test reports showed no growth. ETT changed to $8.5 \mathrm{~mm}$. The patient's independent state on ventilator SIMV needs intensive care and support.

\section{Results}

If any stroke is suspected, immediate evaluation is needed. The examination may reveal evidence of brain injury with weakness, slurred speech, and loss of sensations. Generally, a radiology examination is necessary, such as a CT scan or MRI scan. The CT or MRI can highlight various features and location of brain bleeding. Further testing performed to determine the cause of the bleeding. This additional testing can help to determine if abnormal blood vessels are present as well as the next step in either diagnosis or treatment. In certain situations, a spinal tap (lumbar puncture) advised confirming evidence of bleeding or rule out other brain problems.

\section{Conclusion}

Patients having brainstem hemorrhage resulting in severe brainstem compression and progressive deterioration of clinical features should undergo prompt surgical removal of hematoma, followed by postoperative medical management in a neurosurgical intensive care unit. With the widening knowledge in microsurgical techniques as well as neuroimaging technology, there seems to have more optimistic hope for the surgical management of spontaneous hypertensive brainstem hemorrhage for better prognosis.

\section{References}

1. Drake M, Bernard A, Hessel E. Brain Death. Surg Clin North Am. 2017; 97: 1255-1273.

PubMed: https://www.ncbi.nlm.nih.gov/pubmed/29132508

2. Merai R, Siegel C, Rakotz M. CDC Grand Rounds: A Public Health Approach to Detect and Control Hypertension. MMWR Morb Mortal Wkly Rep. 2016; 65: 1261-1264.

PubMed: https://www.ncbi.nlm.nih.gov/pubmed/27855138

3. Kerber KA, Brown DL, Lisabeth LD, Smith MA, Morgenstern LB. Stroke among patients with dizziness, vertigo, and imbalance in the emergency department: a population-based study. Stroke. 2006; 37: 2484-2487.

PubMed: https://www.ncbi.nlm.nih.gov/pubmed/16946161

4. Teasell R, Foley N, Doherty T, Finestone H. Clinical characteristics of patients with brainstem strokes admitted to a rehabilitation unit. Arch Phys Med Rehabil. 2002; 83: 1013-1016. PubMed: https://www.ncbi.nlm.nih.gov/pubmed/12098164

5. Wardlaw JM, Murray V, Berge E, Del Zoppo GJ. Thrombolysis for acute ischaemic stroke. The Cochrane database of systematic reviews. 2014.

6. Aronow WS. Treatment of hypertensive emergencies. Annals of translational medicine. 2017; 5: 5 . 